\title{
Defect Correction and Multigrid for an Efficient and Accurate Computation of Airfoil Flows*
}

\author{
BARRY KOREN \\ Centre for Mathematics and Computer Science, \\ P.O. Box 4079. 1009 AB Amsterdam, The Netherlands
}

Received August 25, 1986; revised March 18, 1987

\begin{abstract}
Results are presented for an efficient solution method for second-order accurate discretizations of the 2D steady Euler equations. The solution method is based on iterative defect correction. Several schemes are considered for the computation of the second-order defect. In each defect correction cycle, the solution is computed by non-linear multigrid iteration, in which collective symmetric Gauss-Seidel relaxation is used as the smoothing procedure. A finite volume Osher discretization is applied throughout. The computational method does not require any tuning of parameters. The airfoil flow solutions obtained show a good resolution of all flow phenomena and are obtained at low computational costs. The rate of convergence is grid-independent. The method contributes to the state of the art in efficiently computing flows with discontinuities. 1988 Academic Press, Inc.
\end{abstract}

\section{INTRODUCTION}

Recently, a very efficient multigrid method has been developed for the solution of a robust, first-order accurate discretization of the Euler equations [6]. Two wellknown drawbacks of first-order accurate discretizations of the Euler equations are: (i) their need for relatively fine grids in smooth flow regions and (ii) their strong smearing of discontinuities that are not aligned with the grid. Second-order accurate discretizations yield a strong improvement of both drawbacks. However, second-order discretizations cannot be solved with the same efficiency by the multigrid method. Motivated by the requirement of computational efficiency, Hemker [5] and Spekreijse [11] investigated a solution method for second-order schemes. The method is based on iterative defect correction [2].

Iterative defect correction is known to be efficient for smooth problems. In this paper, we show that it is also feasible for the efficient computation of non-smooth problems. We show the behaviour of defect correction iteration for five different second-order schemes. The discussion is restricted to a number of well-known airfoil flow problems from $[10,14]$ : the NACA0012-airfoil at $M_{\infty}=0.63, \alpha=2^{\circ}$; $M_{\star}=0.8, \alpha=1.25^{\circ} ; M_{\infty}=0.85, \alpha=1^{\circ}$, and $M_{\infty}=1.2, \alpha=7^{\circ}$.

* This work was supported by the Netherlands Technology Foundation (STW). 
In Section 2, we briefly describe the basic discretization technique. In Section 3, we discuss the solution method used: non-linear multigrid as an inner iteration for the solution of the elementary first-order system and defect correction as an outer iteration for the solution of the second-order system. In Section 4, we describe the five second-order schemes that were used in our computations. The main results of this paper are given in Section 5. Conclusions are summarized in Section 6.

\section{Discretization}

The steady 2D Euler equations can be written on the domain $\Omega$ as

$$
\frac{\partial f(q)}{\partial x}+\frac{\partial g(q)}{\partial y}=0
$$

with

$$
q=\left(\begin{array}{c}
\rho \\
\rho u \\
\rho v \\
\rho e
\end{array}\right), \quad f=\left(\begin{array}{c}
\rho u \\
\rho u^{2}+p \\
\rho u v \\
\rho u(e+p / \rho)
\end{array}\right), \quad g=\left(\begin{array}{c}
\rho v \\
\rho v u \\
\rho v^{2}+p \\
\rho v(e+p / \rho)
\end{array}\right) \text {, }
$$

and

$$
e=\frac{1}{\gamma-1} \frac{p}{\rho}+\frac{1}{2}\left(u^{2}+v^{2}\right)
$$

Here, $\rho, u, v, p$, and $\gamma$ denote respectively density, velocity components in $x$ - and $y$-directions, static pressure, and ratio of specific heats.

Following [6], we solve the Euler equations in their integral form

$$
\int_{\delta \Omega^{*}}\left(f n_{x}+g n_{y}\right) d s=0
$$

With $\delta \Omega^{*}$ we denote the boundary of an arbitrary subregion $\Omega^{*} \subset \Omega$, and with $n_{x}$ and $n_{y}$ the components of the outward normal with unit length, along $\delta \Omega^{*}$. A simple way to discretize $(2.4)$ is to subdivide $\Omega$ into disjoint quadrilateral subregions $\Omega_{i j}$ (finite volumes) and to assume that the functions $f$ and $g$ are constant along each volume wall and that they are functions of the state at the left and right side of each volume wall only. This gives us the discretization

$$
\sum_{k=1}^{4}\left\{f\left(q_{i j, k}^{1}, q_{i j, k}^{\mathrm{r}}\right) n_{x_{i j, k}}+g\left(q_{i j, k}^{1}, q_{i j, k}^{\mathrm{r}}\right) n_{y_{i j, k}}\right\} s_{i j, k}=0 \quad \text { for all } i j
$$

with $f\left(q^{1}, q^{\mathrm{r}}\right)$ and $g\left(q^{1}, q^{\mathrm{r}}\right)$ the so-called numerical flux functions, $s_{i j, k}$ the length of 
the $k$ th wall of $\Omega_{i j}$, and 1 and $\mathrm{r}$ superscripts referring to the left and right side of a wall, respectively.

For the Euler equations, because of their rotational invariance, (2.5) may be further simplified $[11]$ to

$$
\sum_{k=1}^{4} T_{i j, k}^{-1} f\left(T_{i j, k} q_{i j, k}^{1}, T_{i j, k} q_{i j, k}^{\mathrm{r}}\right) s_{i j, k}=0 \quad \text { for all } i j
$$

with

$$
T_{i j, k}=\left(\begin{array}{cccc}
1 & 0 & 0 & 0 \\
0 & n_{x_{i, k}} & n_{y_{i, k}} & 0 \\
0 & -n_{y_{y, k}} & n_{x_{y, k}} & 0 \\
0 & 0 & 0 & 1
\end{array}\right),
$$

and with $n_{x_{l, k}}$ and $n_{y_{i, k}}$ the components of the outward unit normal on $\partial \Omega_{i j, k}$.

In each volume, we assume the state to be an approximation of the mean value of the exact solution. When we take $q_{i j, k}^{\mathrm{l}}$ and $q_{i j, k}^{\mathrm{r}}$ equal to the states in volume $\Omega_{i j}$ and its neighbouring volume $\Omega_{i j, k},(2.6)$ is first-order accurate only. Second-order accuracy can be obtained in a simple way by determining $q_{i j, k}^{1}$ and $q_{i j, k}^{r}$ as either interpolations or extrapolations by low degree piecewise polynomial functions, using two or three adjacent volume states. Schemes for inter- and extrapolation of volume states are called projection schemes [8].

For the evaluation of the numerical flux vector $f$ at the volume walls, we consider the flow at each volume wall as the local solution of the 1D Riemann problem for the two gas states $q_{i j, k}^{1}$ and $q_{i j, k}^{r}$. For the solution of the 1D Riemann problem, we have chosen the approximate Riemann solver as proposed by Osher [9]. The choice for Osher's scheme is motivated among others by: (i) its consistent treatment of boundary conditions, and particularly (ii) by its continuous differentiability and therefore its suitability for a Newton-type solution technique. (A Newton-type solution technique is used in the relaxation method which is part of the multigrid technique used.)

\section{SOLUTION METHOD}

When one uses the first-order discretization, the non-linear system (2.6) becomes

$$
\sum_{k=1}^{4} T_{i j, k} f\left(T_{i j, k} q_{i j}, T_{i j, k} q_{i j, k}\right) s_{i j, k}=0 \quad \text { for all } i j
$$

in which $q_{i j}$ denotes the state in volume $\Omega_{i j}$, and $q_{i j, k}$ that in the neighbouring volume $\Omega_{i j, k}$.

To solve this system, we already considered $[6,7]$ point $(=$ volume) relaxation 
methods, in which we used one or more local Newton steps for the collective relaxation of the 4 unknowns in each single volume. The most efficient relaxation was obtained by selecting a large tolerance for the Newton iteration, so that in all but exceptional cases only a single Newton step was taken. These relaxation methods are simple and robust, but need an acceleration. When one uses, as in $[6,7]$, collective symmetric Gauss-Seidel as a point relaxation method, a suitable acceleration technique is found in multigrid. As a very efficient and robust multigrid technique we used: the full approximation scheme (FAS), preceded by full multigrid (FMG) to obtain a good initial estimate [4].

However, when one uses a higher order discretization and adopts this solution method, one will severely lose in efficiency. Point Gauss-Seidel will no longer be a good smoother. To circumvent the difficulty of finding a sufficiently good smoother, we use an iterative defect correction (IDeC) process as solution method [2]. Denoting the system of equations resulting from a first- and second-order discretization as $N_{h}^{1}\left(q_{h}\right)=0$ and $N_{h}^{2}\left(q_{h}\right)=0$, respectively, the IDeC-process can be written as

$$
\begin{gathered}
N_{h}^{1}\left(q_{h}^{1}\right)=0, \\
N_{h}^{1}\left(q_{h}^{n+1}\right)=N_{h}^{1}\left(q_{h}^{n}\right)-N_{h}^{2}\left(q_{h}^{n}\right), \quad n=1,2, \ldots, N
\end{gathered}
$$

As solution method for $N_{h}^{1}\left(q_{h}\right)=r_{h}$ as it appears in both (3.2a) and (3.2b), we maintain the efficient multigrid method just described.

It is well known [4] that if the problem is smooth enough, $q_{h}^{2}$ is second-order accurate already. If the solution is non-smooth, i.e., when higher derivatives are dominating, there is no reason to expect $q_{h}^{2}$ to be more accurate than $q_{h}^{1}$. Nevertheless, in Section 5 evidence is given that for non-smooth problems, a single IDeC-cycle may improve the solution significantly. In fact we may use $q_{h}^{n+1}-q_{h}^{n}$ as an error indicator. In the smooth parts of the solution $q_{h}^{2}-q_{h}^{1}=O(h)$, and $q_{h}^{3}-q_{h}^{2}=O\left(h^{2}\right)$. Where these differences are larger, e.g., $O(1)$, the solution is nonsmooth (relative to the grid used). There, if a more accurate solution is wanted, grid refinement is to be considered rather than a higher order discretization.

\section{Projection SChemes}

As standard projection schemes, we consider the central, the upwind and an upwind biased scheme. Let $q_{i+1 / 2, j}^{1(k)}$ and $q_{i+1 / 2, j}^{\mathrm{r}(k)}$ be the $k$ th component $(k=1,2,3,4)$ of $q_{i+1 / 2, j}^{1}$ and $q_{i+1 / 2, j}^{\mathrm{r}}$, in which $i+\frac{1}{2}$ refers to the wall separating volume $i$ and $i+1$. Then these three schemes can be written as

$$
\begin{aligned}
& q_{i+1 / 2, j}^{1(k)}=q_{i, j}^{(k)}+\frac{1+\kappa}{4}\left(q_{i+1, j}^{(k)}-q_{i, j}^{(k)}\right)+\frac{1-\kappa}{4}\left(q_{i, j}^{(k)}-q_{i-1, j}^{(k)}\right), \\
& q_{i+1 / 2, j}^{\mathrm{r}(k)}=q_{i+1, j}^{(k)}+\frac{1+\kappa}{4}\left(q_{i, j}^{(k)}-q_{i+1, j}^{(k)}\right)+\frac{1-\kappa}{4}\left(q_{i+1, j}^{(k)}-q_{i+2, j}^{(k)}\right) .
\end{aligned}
$$


For $\kappa=1, \kappa=-1$, and $\kappa=\frac{1}{3}$, we get the central, the upwind, and the upwind biased schemes, respectively. Similar relations hold for $q_{i, j+1 / 2}^{1(k)}$ and $q_{i, j+1 / 2}^{r(k)}$. A property of the central scheme is that it makes the Riemann solver superfluous. Properties of all three schemes are: (i) that they cannot be applied in a consistent way in the neighbourhood of boundaries, and (ii) that they may yield solutions with spurious non-monotonicity (wiggles). In the context of IDeC, wiggles and even instability might not be serious problems. They might become significant in a later stage of the process only, a stage not to be reached in practice.

As a projection scheme which is consistent near boundaries, Hemker [5] introduced the so-called superbox scheme. A superbox is defined as a set of $2 \times 2$ volumes. At the 4 inner walls of a superbox the simple central projection scheme is used, whereas at the 8 outer walls the upwind scheme is used. A property of the superbox scheme is that its solutions are second-order accurate per arbitrary set of $2 \times 2$ volumes, but not per single volume. The remaining lower order error contains only high frequencies. This error can be eliminated in a simple way by computing states at volume vertices as averages over neighbouring volumes. (Formal proofs can be given.) Similar to the aforementioned schemes, the superbox scheme may also yield solutions with wiggles.

If a monotone solution is required, and if it might be necessary for some problem to suppress wiggles indeed, the question arises whether it is suitable to use a flux limiter $[12,13]$ for this purpose. Projection schemes using flux limiters can be written as

$$
\begin{aligned}
& q_{i+1 / 2, j}^{\mathrm{l}(k)}=q_{i, j}^{(k)}+\frac{1}{2} \psi\left(R_{i, j}^{(k)}\right)\left(q_{i, j}^{(k)}-q_{i-1, j}^{(k)}\right), \\
& q_{i+1 / 2, j}^{\mathrm{r}(k)}=q_{i+1, j}^{(k)}+\frac{1}{2} \psi\left(\frac{1}{R_{i+1, j}^{(k)}}\right)\left(q_{i+1, j}^{(k)}-q_{i+2, j}^{(k)}\right),
\end{aligned}
$$

with $\psi$ denoting the flux limiter, and with

$$
R_{i, j}^{(k)}=\frac{q_{i+1, j}^{(k)}-q_{i, j}^{(k)}}{q_{i, j}^{(k)}-q_{i=1, j}^{(k)}}
$$

We prefer a limiter which is smooth and which renders a scheme at the upstream side of shock waves which is close to the upwind scheme (a natural scheme in those regions). To satisfy these requirements we can simply use the van Albada limiter [1], which is defined by

$$
\psi(R)=\frac{R^{2}+R}{R^{2}+1}
$$

Projection schemes using flux limiters need (at least) three volume states per projec- 
tion. This implies that they cannot be used in a consistent way near boundaries. Near boundaries one has to use other schemes, as for instance the central and the upwind scheme, which may both introduce some small wiggles.

\section{Results}

For all airfoil flows considered, we used $O$-type grids with the outer boundary at an approximate distance from the airfoil of either 25 or 100 chord lengths. For both distances, we imposed the unperturbed flow at the outer boundary, although we did not overimpose. (I.e., for, e.g., a subsonic outer boundary, we did not impose more than 3 boundary conditions at the inlet part of that boundary and not more than 1 boundary condition at the outlet part.)

For each of the projection schemes considered, the Kutta condition was automatically satisfied for all flows. For all flows a streamline smoothly left the trailing edge. An explanation for the fact that there is no flow around the airfoil's tail may be the property of all discretizations that they are non-isentropic. Along both the upper and lower airfoil surface, they all generate spurious changes of total pressure. As a consequence, a flow around the airfoil's tail would in general result in a stagnation at two different pressures, which is an unstable flow situation.

At first, we investigated the iterative solution method for the non-linear systems (3.2a) and (3.2b) (inner iteration). It was found that the convergence rate is independent of the starting point of the relaxation sweeps. However, we found that for efficient smoothing one should always make symmetric sweeps. So, for instance, if one starts at the airfoil, one should not stop at the outer boundary, but return from the outer boundary to the airfoil and stop there.
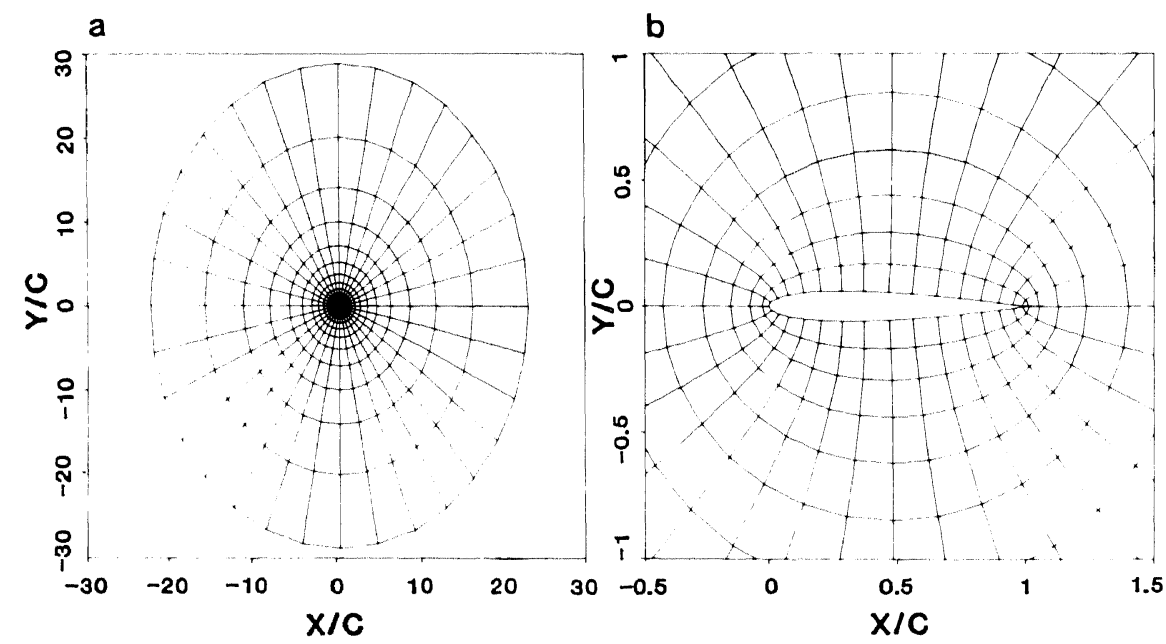

FIG. 5.1. $32 \times 16$-grid, NACA0012-airfoil: (a) in full; (b) in detail. 
Concerning the multigrid strategy, it appeared that the straightforward use of $V$-cycles with a single symmetric pre- and post-relaxation per level gives the best convergence rate. For all results presented in this paper, we used a coarsest grid with 8 volumes tangentially to the airfoil and either 4 or 2 volumes radially. We notice that this is extremely coarse, if seen in the light of suspicions raised by Eriksson and Rizzi [3] against the possibilities of a multigrid method.

To study the behaviour of IDeC for the various projection schemes, we considered as standard test case: the NACA0012-airfoil at $M_{x}=0.8, \alpha=1.25^{\circ}$ (transonic flow with shock).

First, to investigate the convergence properties of $\mathrm{IDeC}$ for the various projection schemes, we performed for each scheme $10 \mathrm{IDeC}$-cycles, with $5 \mathrm{FAS}$-cycles per IDeC-cycle. As finest grid, we used a moderately stretched $32 \times 16$-grid (Fig. 5.1), yielding a 3-level multigrid strategy.

In Fig. 5.2a, convergence histories are given by graphs of the residual ratio $\sum_{i=1}^{4}\left|\bar{r}_{h}^{n}(i)\right| / \sum_{i=1}^{4}\left|\bar{r}_{h}^{1}(i)\right|$ versus the number of FAS-cycles. Here, $\bar{r}_{h}^{n}$ denotes the summation over all volumes of $r_{h}^{n}=N_{h}^{2}\left(q_{h}^{n}\right), i$ the $i$ th residual component, and $n$ the $n$th iterand in (3.2). As a starting point in the convergence histories, the first-order solution obtained from (3.2a) is used. The vertical lines mark the beginnings and ends of the IDeC-cycles. In Fig. 5.2b, graphs are given of the surface distribution of the entropy ratio $s / s_{x}$, with $s=p \rho^{-}{ }^{*}$. For this, the curves with circular markers indicate the upper surface distributions, whereas the curves with triangular markers indicate the lower surface distributions. Except for the superbox scheme, the markers correspond with the $x$-locations of the volume wall centres at the airfoil's surface. (For the superbox scheme, due to the averaging, they correspond with the volume wall vertices at the airfoil's surface.)

It appears that both the central and upwind scheme lead to an early divergence of IDeC. For the central scheme, the shock behaves as source of instability. For the upwind scheme, the stagnation region behaves as such. The instability of IDeC for the upwind scheme can be explained by applying local model analysis to the linear convection equation.

The upwind biased scheme, the superbox scheme, and the upwind scheme supplied with the van Albada limiter (van Albada scheme) all give convergence. Their convergence histories suggest that the use of 1 or 2 FAS-cycles per IDeC-cycle is sufficient. To investigate the optimal number of FAS-cycles per IDeC-cycle, we performed for the converging schemes successively: 20 IDeC-cycles with 1 FAScycle per IDeC-cycle, and $10 \mathrm{IDeC}$-cycles with $2 \mathrm{FAS}$-cycles per IDeC-cycle. As finest grid, we used again the $32 \times 16$-grid as shown in Fig. 5.1.

The convergence histories obtained are given in Fig. 5.3. As starting point, we used again the first-order solution obtained from (3.2a). It clearly appears from Fig. 5.3 that the strategy with 1 FAS-cycle per IDeC-cycle is most efficient for each of the three projection schemes considered.

Next, we compared some qualitative properties of the fully converged solutions of the first-order, upwind biased, superbox, and van Albada scheme. As finest grid, we used again the $32 \times 16$-grid. To be sure that the solutions were fully converged, 
a
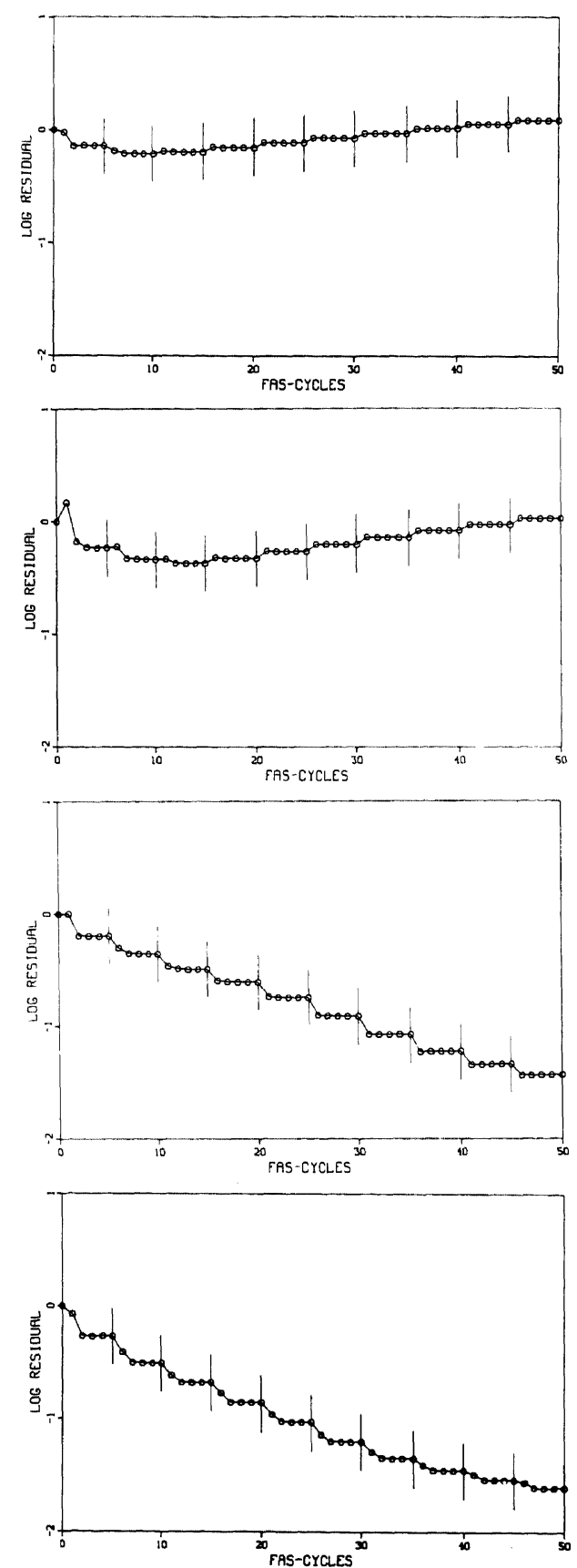

b

Central.

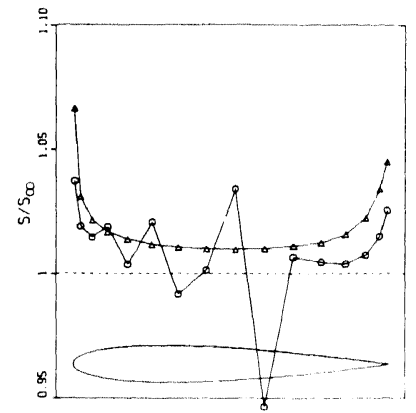

Upaind.
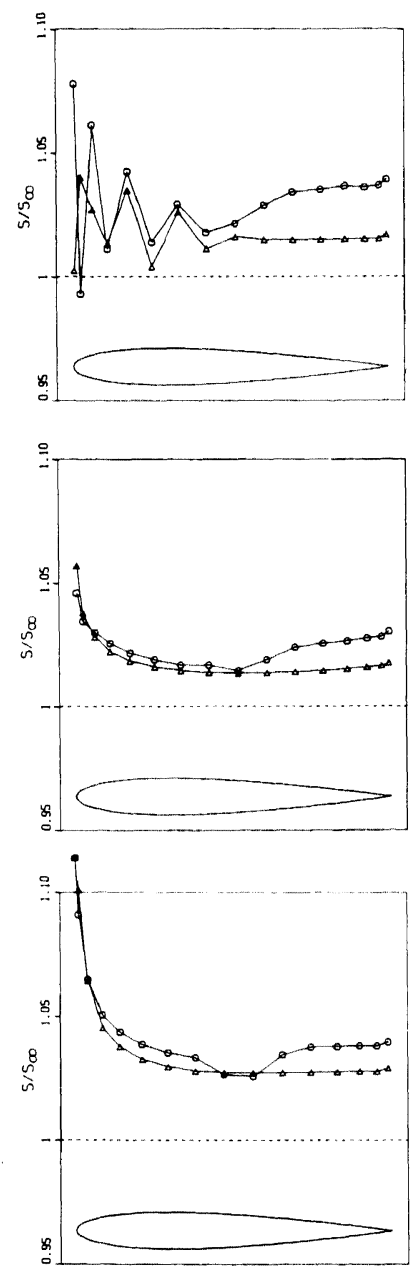

FIG. 5.2. Results of second-order schemes (NACA0012, $M_{x}=0.8, \alpha=1.25^{\circ}$ ): (a) convergence histories; (b) surface entropy distributions. 
a

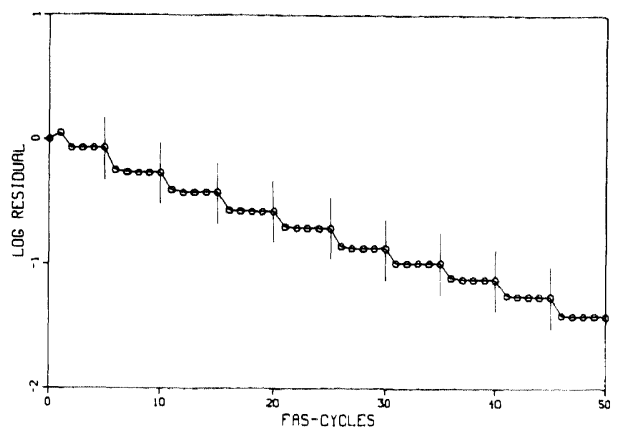

b

van Albada

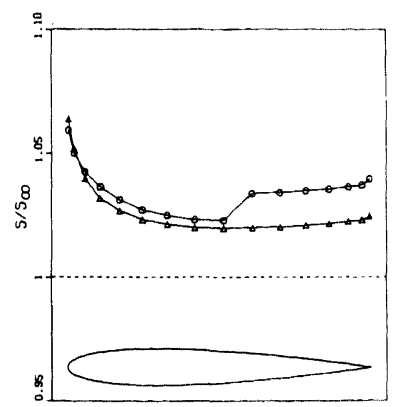

Ficiure $5.2-$ Continued

we used 10 FAS-cycles for the first-order scheme, and 50 IDeC-cycles with 1 FAScycle per IDeC-cycle for the three second-order schemes.

The pressure distributions obtained are given in Fig. 5.4. In each graph, the upper dashed line indicates the critical pressure, and the lower the stagnation pressure. The meaning of the markers is the same as in Fig. 5.2b. Clearly visible in Fig. 5.4 is the strong under- and overshoot at the shock wave, as obtained with the upwind biased and superbox scheme. The small wiggles upstream of the shock, generated by the van Albada scheme must be due to the central and upwind projection that were used near boundaries. Compared with the first-order scheme, all three secondorder schemes give an improvement of the stagnation pressure.

When we take monotonicity at full convergence as a requirement to be fulfilled, only the first-order and van Albada scheme can be used. However, since only a few IDeC-cycles might be necessary (and are desired of course), the question arises how
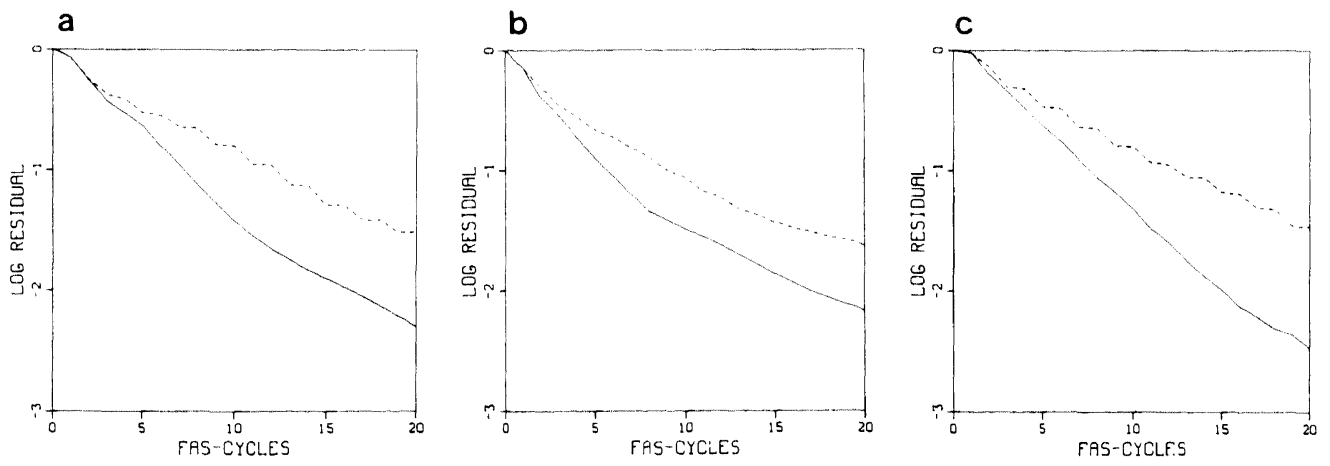

FIG. 5.3. Convergence histories for 1 FAS-cycle per IDeC-cycle (solid) and 2 FAS-cycles per IDeCcycle (dashed), (NACA0012, $M_{t}=0.8, \alpha=1.25$ ): (a) upwind biased; (b) superbox; (c) van Albada. 


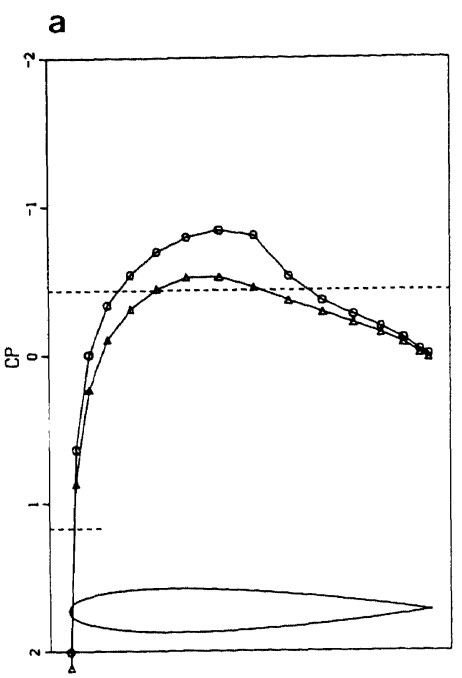

\section{b}

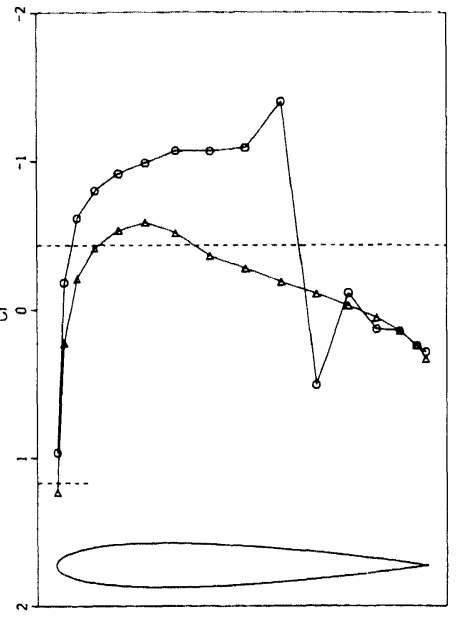

C

d
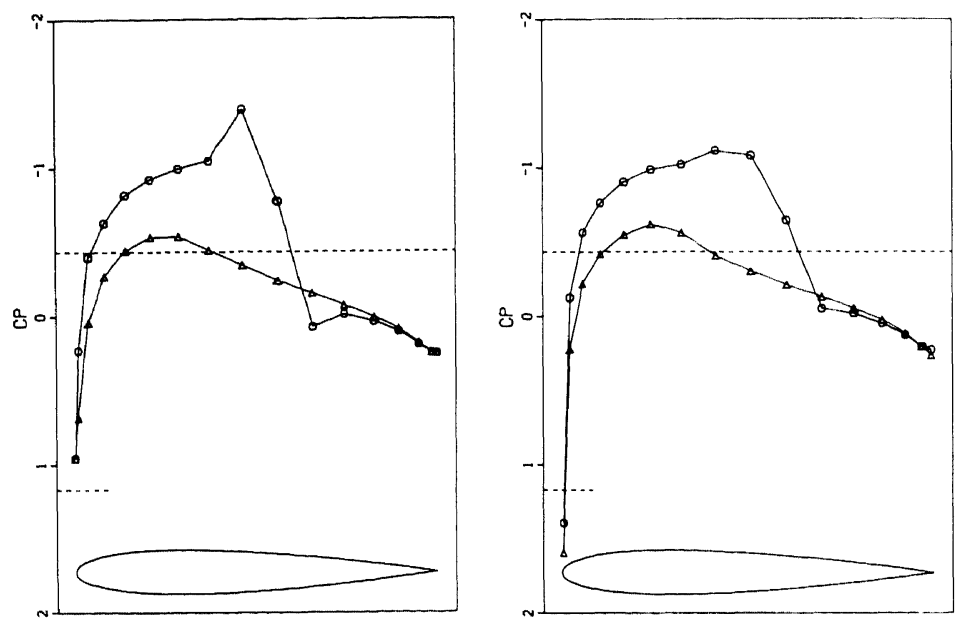

FIG. 5.4. Converged surface pressure distributions (NACA0012, $M,=0.8, \alpha=1.25$ "): (a) first-order; (b) upwind biased; (c) superbox; (d) van Albada.

spurious non-monotonicity at the shock develops in the first IDeC-cycles. To investigate this, we recomputed the flow for both the first-order scheme and the three second-order schemes. As finest grid, we now used a similar, but four times finer grid; a $128 \times 64$-grid (Fig. 5.5). As IDeC-strategy, we used 10 IDeC-cycles with 1 FAS-cycle per IDeC-cycle.

The results obtained are given in Figs. 5.6-5.8. Figures 5.6a and b show the firstorder pressure distribution, as obtained after 1 and 10 FAS-cycles, respectively. The 

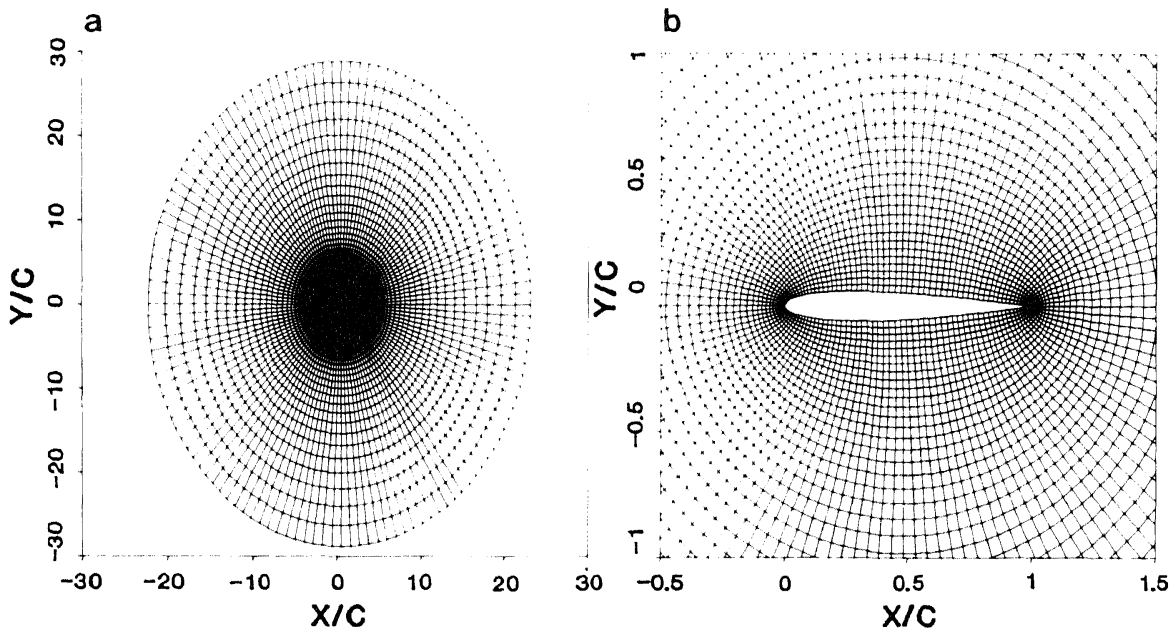

FIG. 5.5. 128 $\times$ 64-grid, NACA0012-airfoil: (a) in full; (b) in detail.

first pressure distribution (Fig. 5.6a) shows, in fact, the first-order solution which is used as initial estimate for (3.2b). The second one (Fig. 5.6b) is that of the fully converged first-order solution. The meaning of the markers and dashed lines in the graphs is the same as before. Figure 5.7 shows for the three second-order schemes the pressure distribution, as obtained after successively the 1st, 2nd, 3rd, and 10th IDeC-cycle. Figure 5.8 shows surface entropy distributions.

From Fig. 5.7, an opposite behaviour after the 1st IDeC-cycle becomes clear. The small wiggles, as obtained with all three schemes after the 1st IDeC-cycle, grow in the following IDeC-cycles for the upwind biased and superbox scheme, but shrink
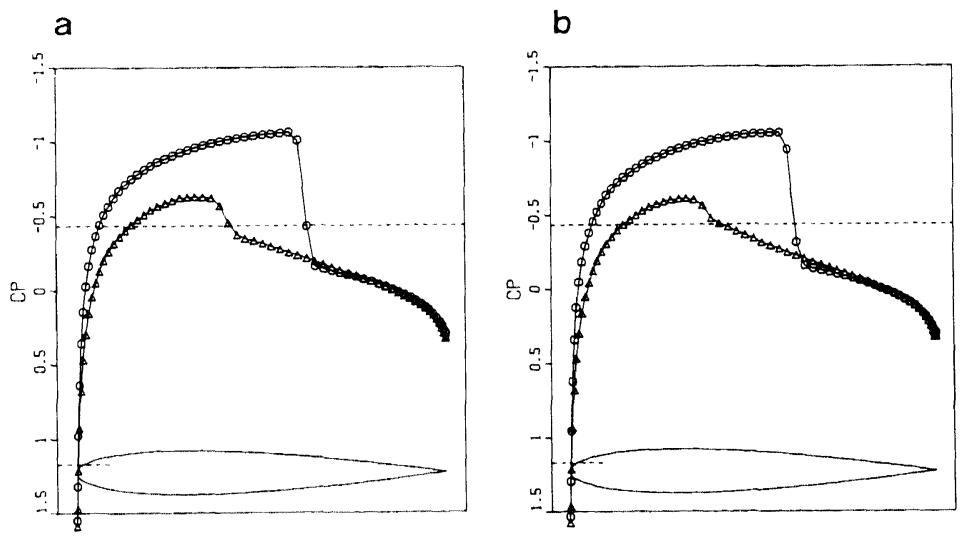

FIG. 5.6. First-order surface pressure distributions (NACA0012, $M_{x}=0.8, \alpha=1.25$ ): (a) after I FAS-cycle; (b) after 10 FAS-cycles. 

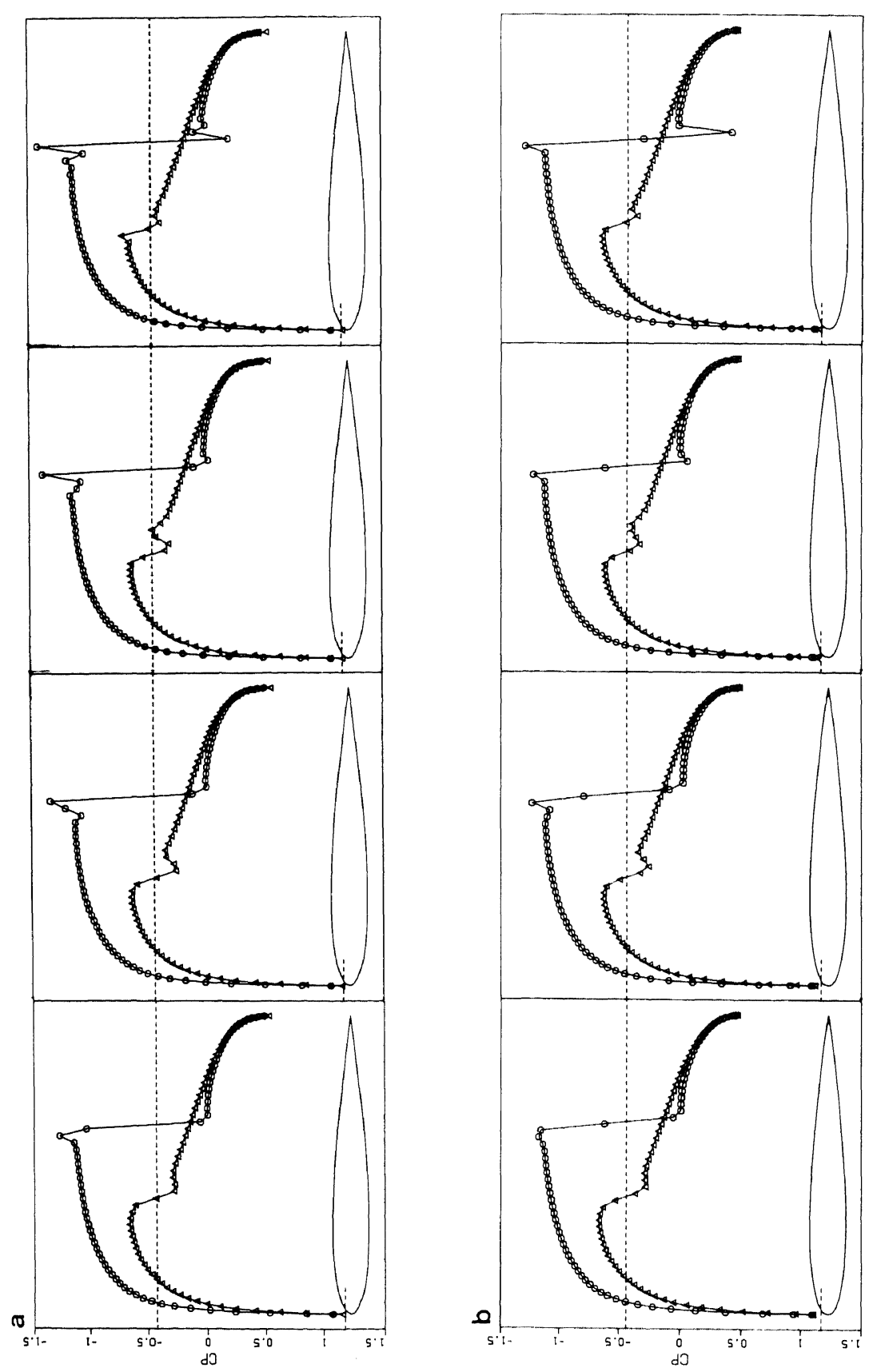


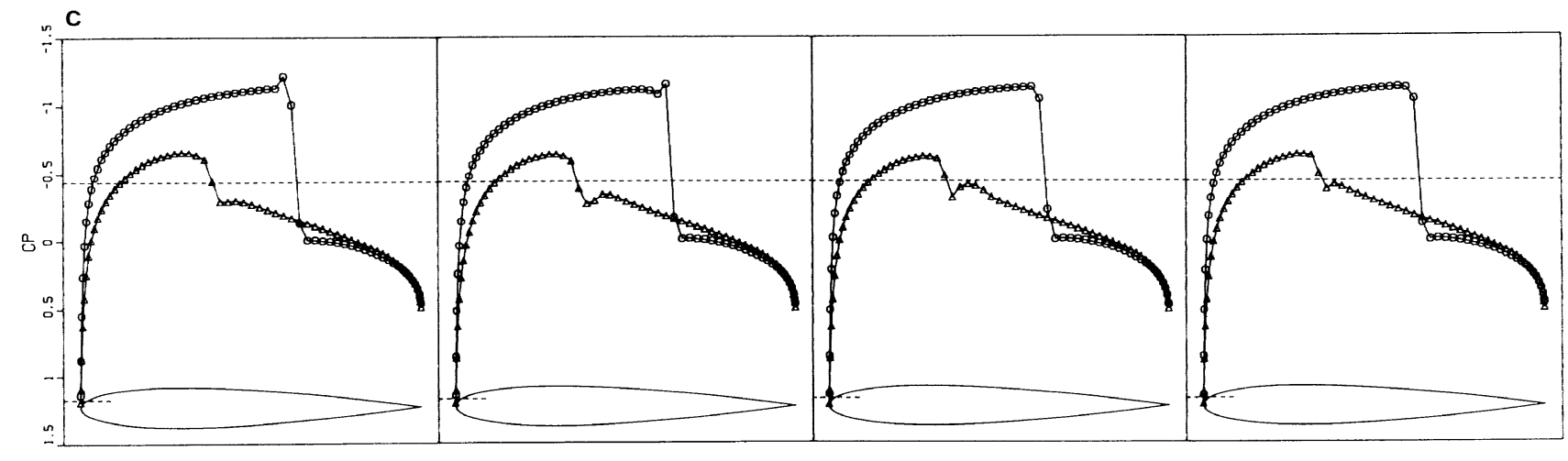

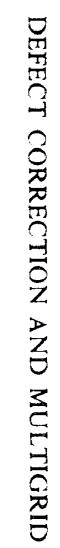

FiG. 5.7. Convergence histories surface pressure distribution second-order schemes (NACA0012, $M_{x}=0.8, x=1.25$ ), after 1st, 2nd, 3rd, and 10th IDeC-cycle: (a) upwind biased; (b) superbox; (c) van Albada. 
for the van Albada scheme. The van Albada scheme yields a nearly wiggle-free solution after the $3 \mathrm{rd}$ IDeC-cycle. Remarkable for all three schemes is the excellent improvement of the stagnation pressure which is obtained in the 1st IDeC-cycle (Fig. 5.6a and 5.7).

In Fig. 5.8, all three second-order schemes show an excellent improvement of the entropy distribution. The upwind biased scheme shows the best improvement.

In Fig. 5.9, we make a comparison with computational results obtained by other investigators, for the transonic flow with shock. In Fig. 5.9a, the left graph shows the pressure distributions that we obtained after $10 \mathrm{IDeC}$-cycles, whereas the right graph stems from [10]. In Fig. 5.9b, we did the same, using the best results from [14].

In Fig. 5.9a, the agreement between the various reference results is poor. This is partly caused by wiggles, but mostly by a large scattering in shock position. This

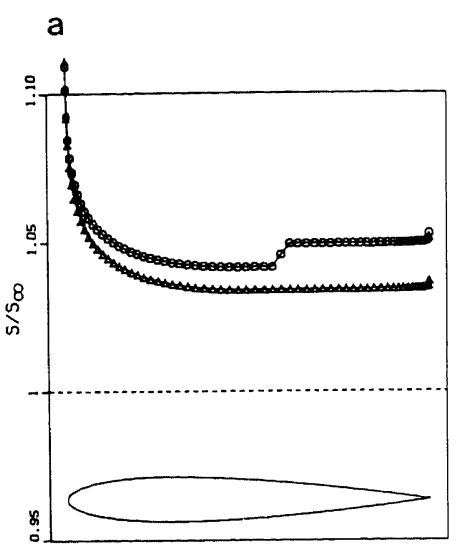

b
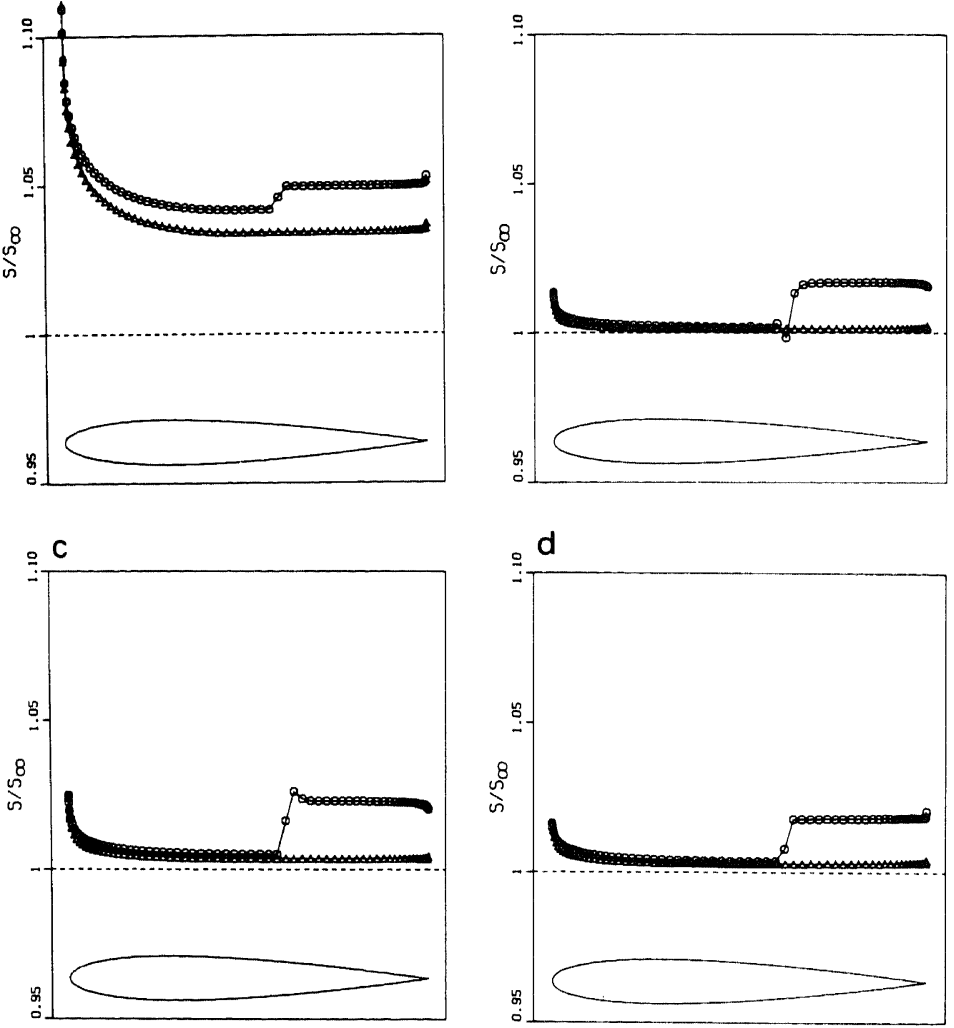

FIG. 5.8. Surface entropy distributions after 10th FAS-cycle (first-order scheme) and 10th IDeCcycle (second-order schemes), (NACA0012, $M_{x}=0.8, \alpha=1.25$ ): (a) first-order; (b) upwind biased; (c) superbox; (d) van Albada. 

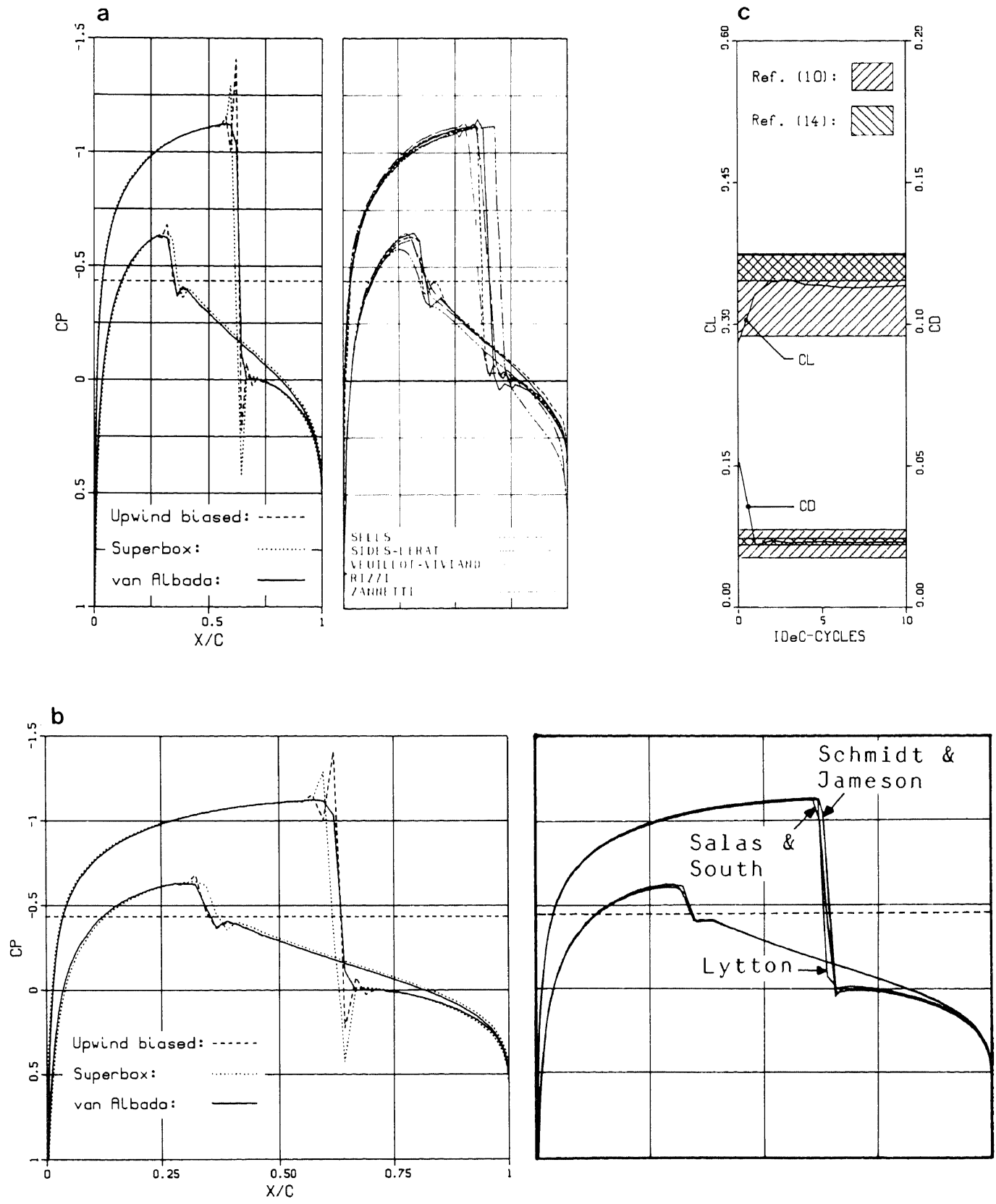

FIG. 5.9. Results for NACA0012-airfoil at $M_{x}=0.8$ and $\alpha=1.25^{\circ}$ : (a) surface pressure distributions: present results (left) and reference results (right); (b) surface pressure distributions: present results (left) and reference results (right); (c) convergence history lift and drag coefficient (van Albada scheme). 

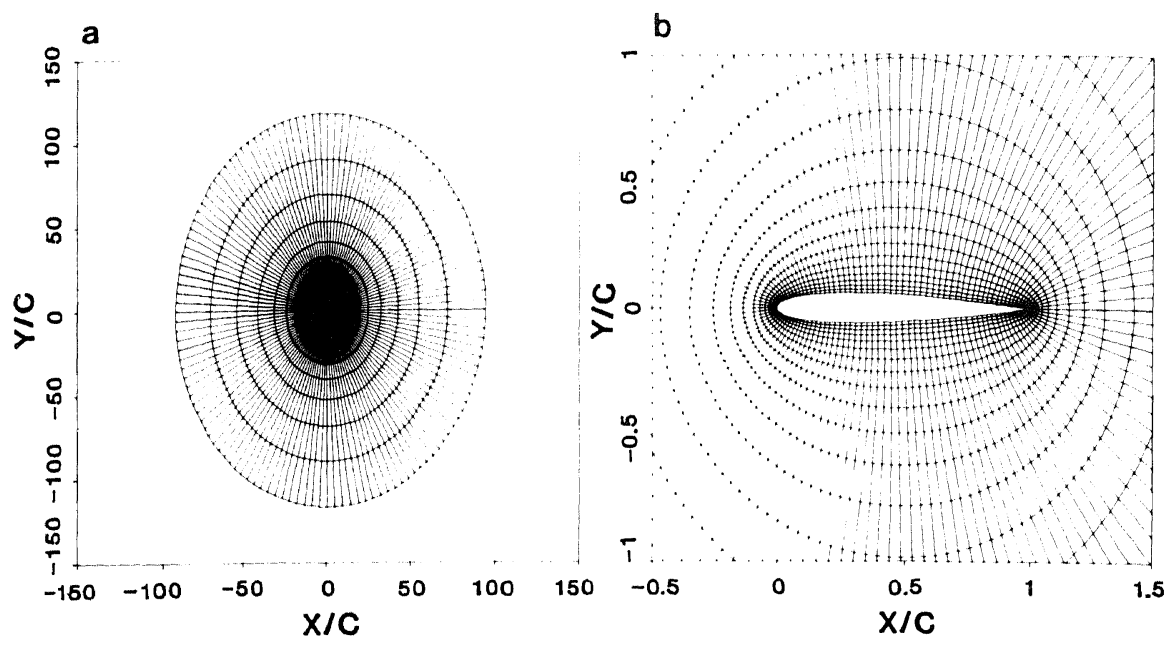

FlG. 5.10. $128 \times 32$-grid: (a) in full; (b) in detail.
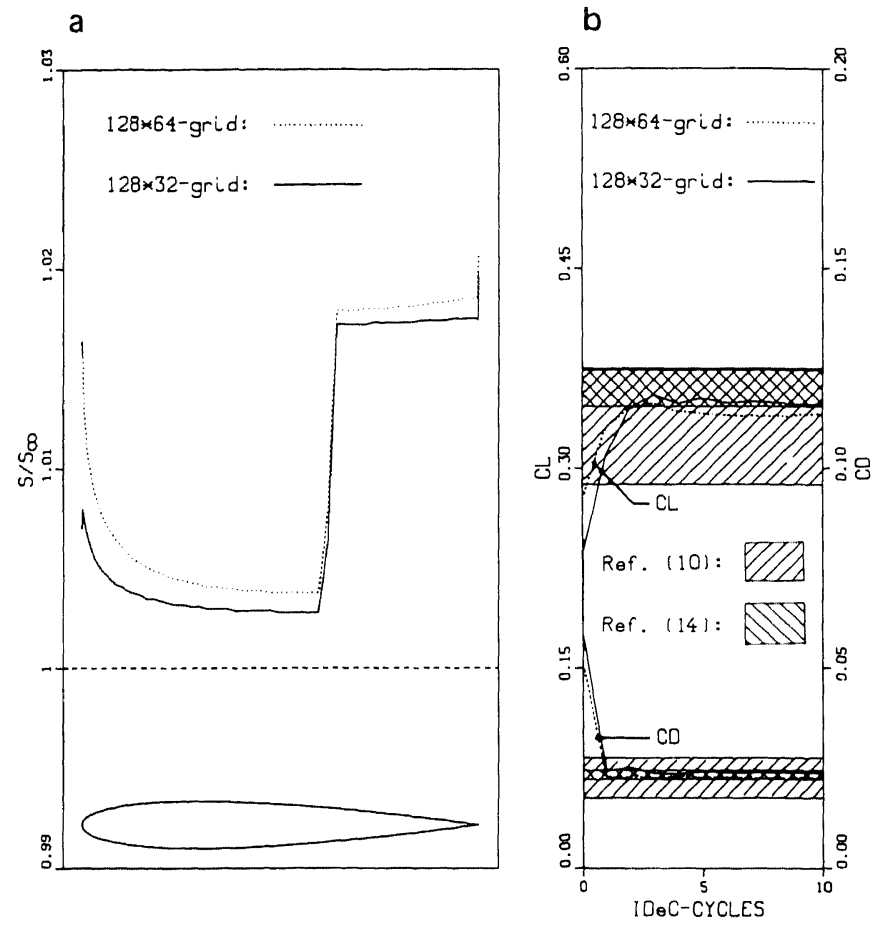

FIG. 5.11. Results of $128 \times 64$ - and $128 \times 32$-grids for NACA0012-airfoil at $M_{,}=0.8$ and $\alpha=1.25^{\circ}$ (van Albada scheme): (a) upper surface entropy distributions; (b) convergence histories lift and drag coefficient. 
scattering is smaller in the more recent reference results of Fig. 5.9b. The agreement between our results and these reference results is good. However, the under- and overshoots, as generated by the upwind biased and superbox scheme, are more severe than those of any of the reference results. The van Albada scheme appears to be most suitable for the computation of flows with shock(s).

In Fig. 5.9c, we present for the van Albada scheme the convergence history of the lift and drag coefficient. As a starting point, we again took the solution obtained from (3.2a). The lift and drag as computed by the other investigators are spread over the shaded areas. One shading represents all (5) Euler results from [10], the other shading all (7) Euler results from [14].

Clearly visible in Fig. 5.9c, is the excellent improvement of the drag, which is obtained in the first IDeC-cycle. (The main cause of this is the strong improvement of the stagnation pressure in the first IDeC-cycle.) When we take the results from [10] as a standard, we see that we only need 1 IDeC-cycle to reach the standard. With the results from [14] as a standard, we end up with a lift which is slightly too
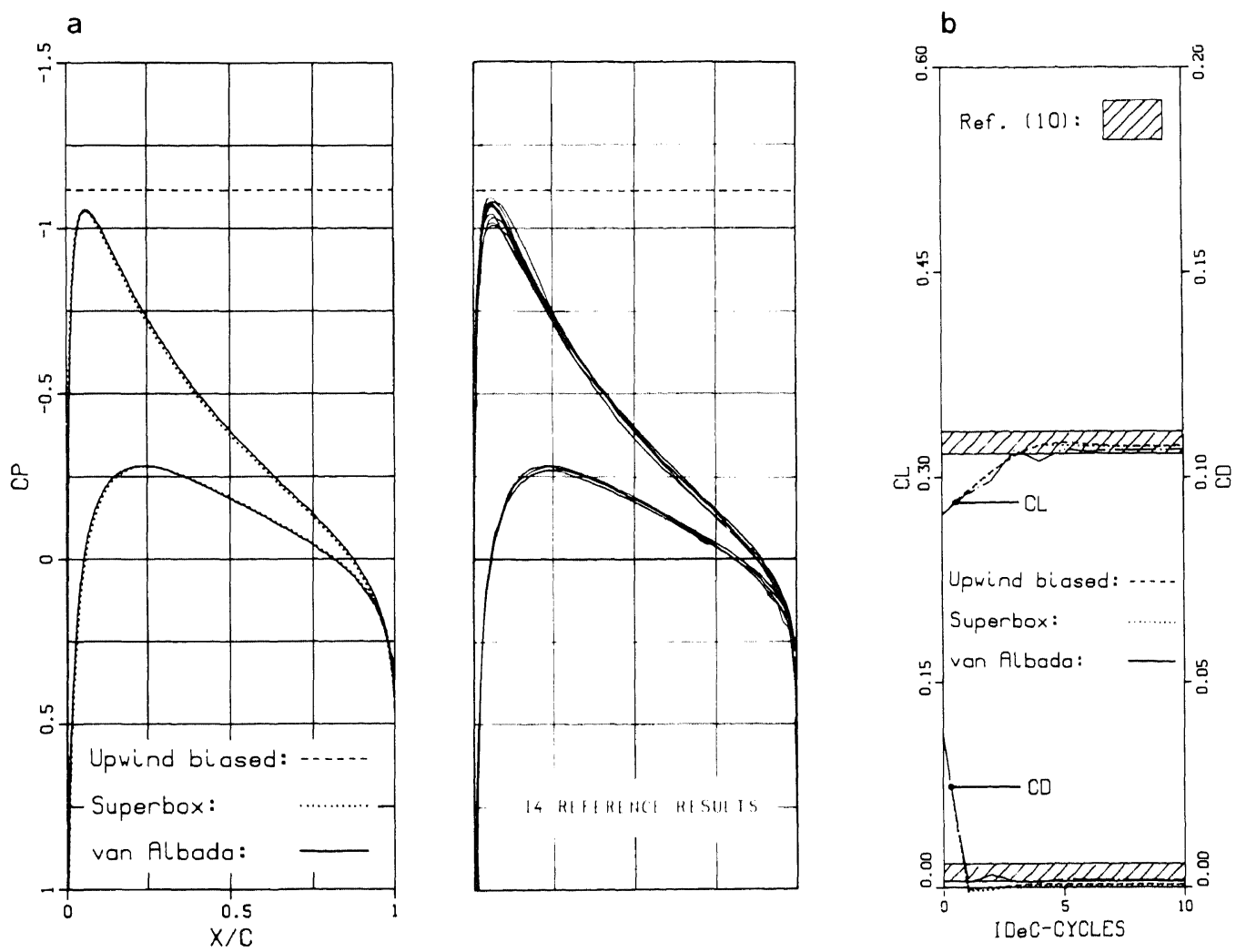

FIG. 5.12. Results for NACA0012-airfoil at $M_{\gamma}=0.63$ and $\alpha=2^{\circ}$ : (a) surface pressure distributions: present results (left) and reference results (right); (b) convergence histories lift and drag coefficient. 

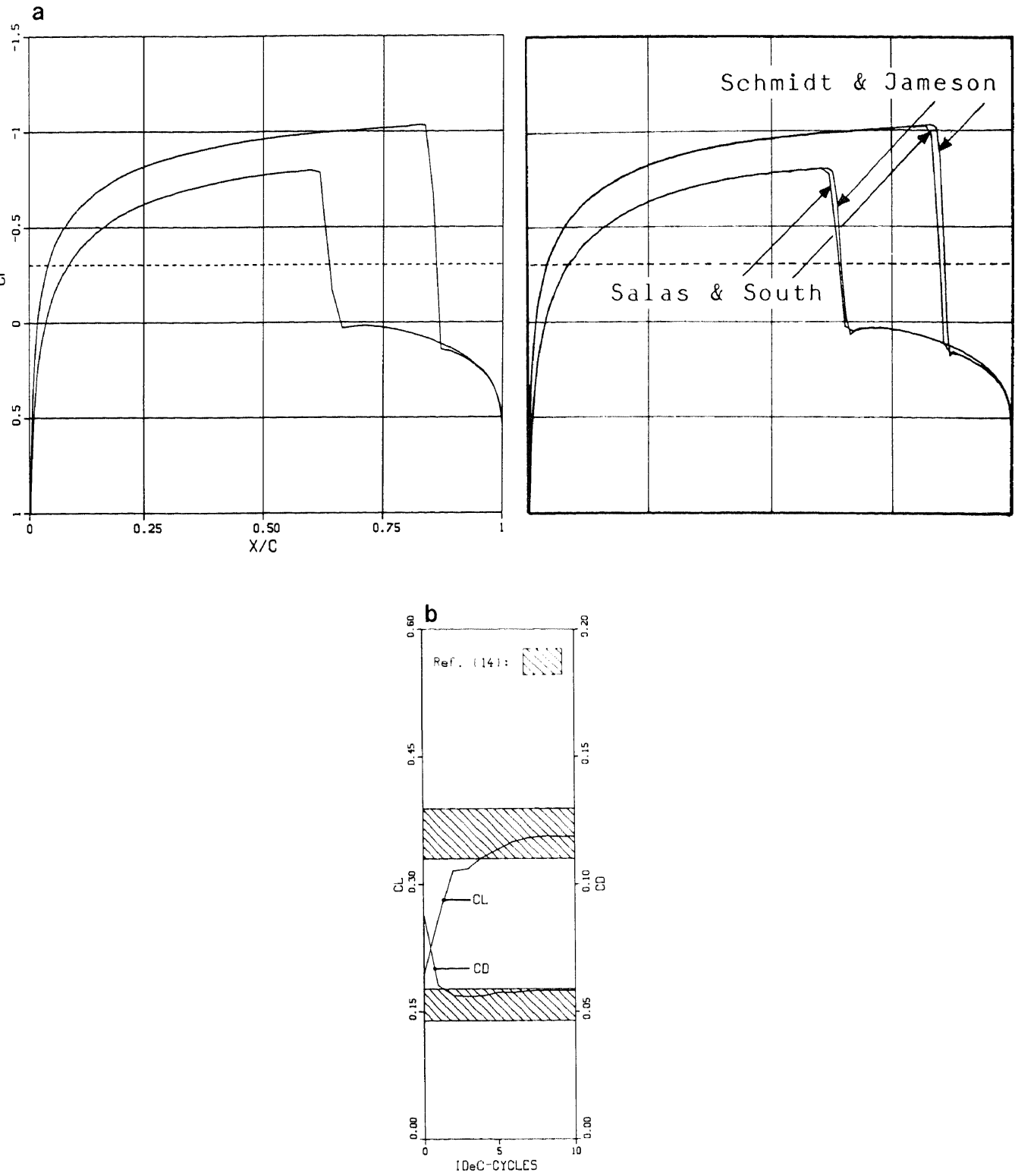

FIG. 5.13. Results for NACA0012-airfoil at $M_{\infty}=0.85$ and $\alpha=1^{\circ}$ (van Albada scheme): (a) surface pressure distributions: present result (left) and reference results (right); (b) convergence history lift and drag coefficient; (c) Mach number distributions: present result (left) and result Schmidt and Jameson (right); (d) present entropy distribution $\left(s / s_{\infty}\right)$. 

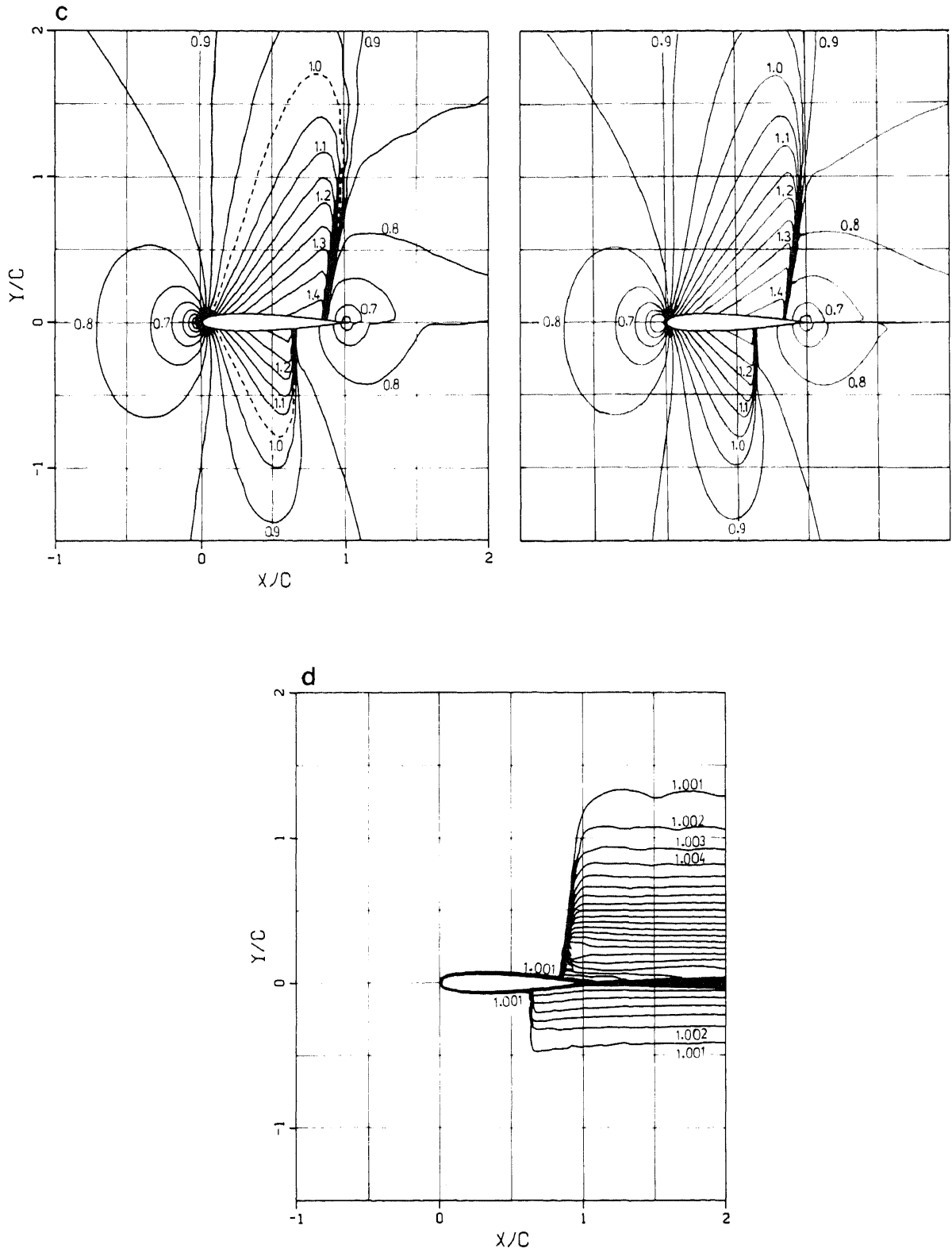

FIGURE 5.13-Continued 
a
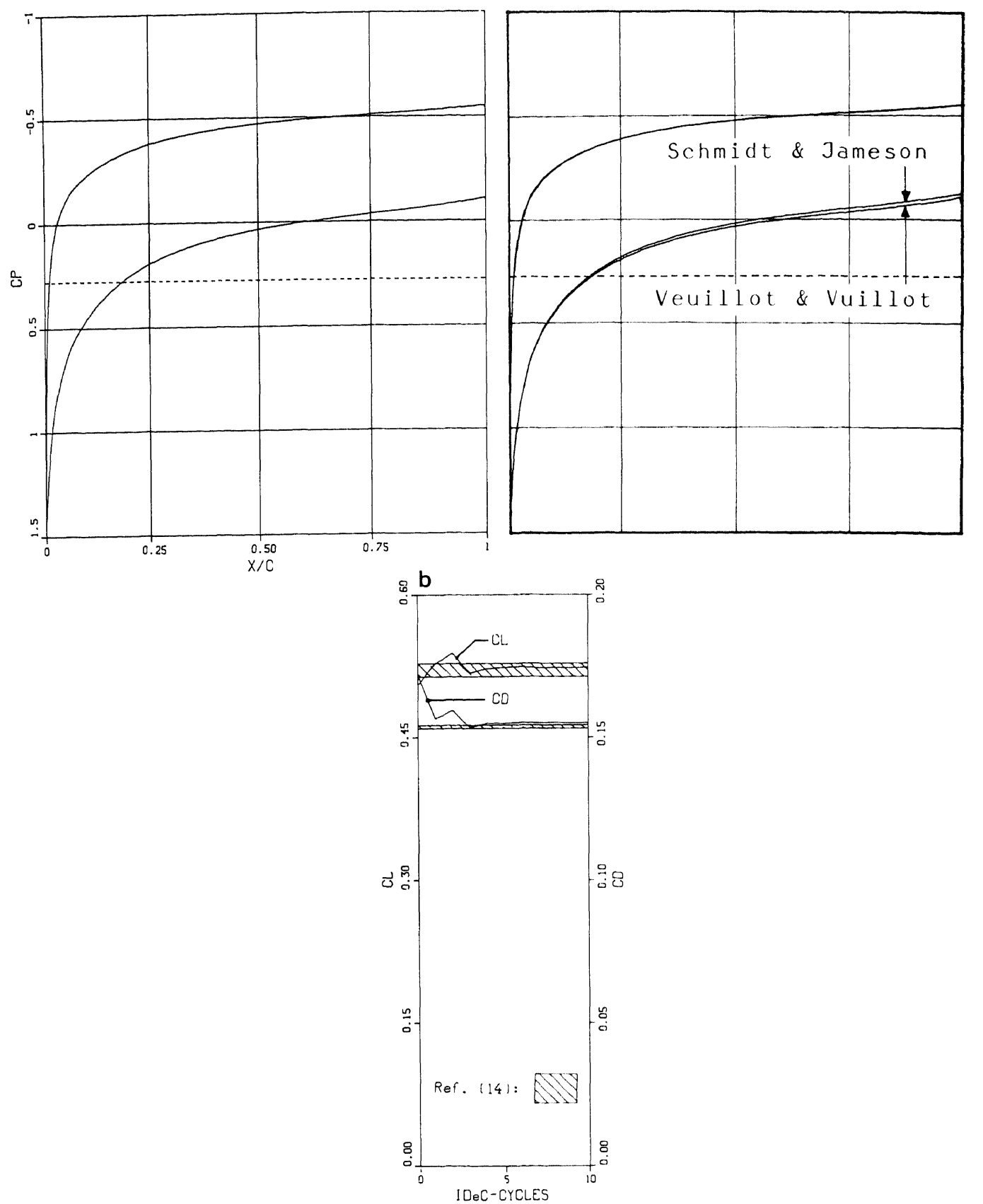

FIG. 5.14. Results for NACA0012-airfoil at $M_{x}=1.2$ and $\alpha=7$ (van Albada scheme). (a) surface pressure distributions: present result (left) and reference results (right); (b) convergence history lift and drag coefficient; (c) Mach number distributions: present result (left) and result Veuillot and Vuillot (right); (d) present entropy distribution $\left(s / s_{x}\right)$. 

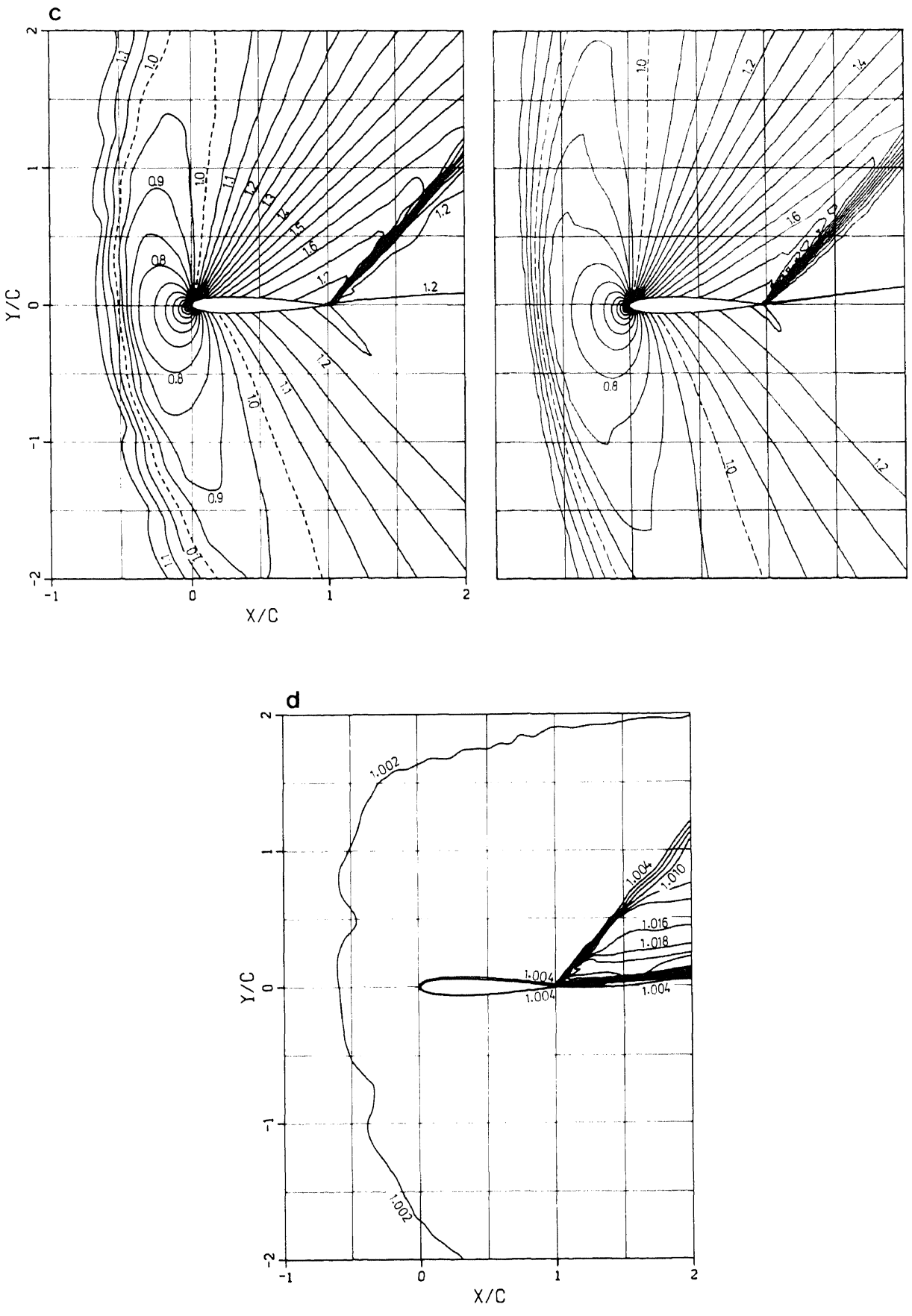

Figure 5.14-Continued 
low. The cause of this discrepancy is thought to be the fact that the outer boundary is not far enough ( $\sim 25$ chord lengths) from the airfoil. We generated a new fine grid (Fig. 5.10), with a twice smaller number of volumes in the radial direction (32 instead of 64 ), but yet with an outer boundary at $\sim 100$ chord lengths, and a twice smaller volume height at the airfoil. Results of a computation with this new grid are given in Fig. 5.11. The improvements are evident. (Concerning the entropy error (Fig. 5.11a), an arbitrary good improvement seems to be possible.)

In Figs. 5.12-5.14, we present comparisons for some other standard problems. As reference results for the lift and drag histories, we again used all Euler results available from either [ 10 or 14$]$, whereas as reference results for the distribution of some solution component, we only selected the best results from either [10 or 14].

As extra test cases we considered the NACA0012-airfoil at: (i) $M_{x}=0.63, \alpha=2^{\circ}$ (subcritical flow); (ii) $M_{x}=0.85, \alpha=1^{\circ}$ (transonic flow with upper shock, lower shock and slip line); and (iii) $M_{x}=1.2, \alpha=7^{\circ}$ (supersonic flow with detached bow shock, oblique tail shock, and slip line). For all three cases, we used as finest grid: the $128 \times 32$-grid shown in Fig. 5.10, and as IDeC-strategy: $10 \mathrm{IDeC}$-cycles with 1 FAS-cycle per IDeC-cycle. As projection scheme for the subcritical test case, we used the upwind biased, the superbox, as well as the van Albada scheme. For the transonic and supersonic test case, we only used the van Albada scheme.

Remarkable in the results obtained for the subcritical test case is the excellent agreement between the surface pressure distributions of the three second-order schemes (Fig. 5.12a). The agreement between our results and the reference results $[10]$ is good. Very good is the drag yielded by the upwind biased and superbox scheme (Fig. 5.12b). Both closely approach the exact zero-drag. The convergence of all three schemes is fast. Within a few IDeC-cycles their corresponding lift and drag values seem to be converged for practical purposes.

For the transonic test case, we compare our solution with those of Schmidt and Jameson [14] and Salas and South [14]. As grid, they used an $O$-type grid of $320 \times 64$ respectively $192 \times 39$. Although no evidence can be given that they both needed such a fine grid, it can be seen that we can use a significantly coarser grid. Our lift and drag agree well with theirs. (Salas and South found: $c_{1}=0.3472$, $c_{\mathrm{d}}=0.0557$, and Schmidt and Jameson found: $c_{1}=0.3584, c_{\mathrm{d}}=0.0580$, whereas we found: $c_{1}=0.3565, c_{\mathrm{d}}=0.0582$ ). Further, all three discontinuities occurring in the flow are captured equally well in our results and the reference results (Fig. 5.13a and c). Our entropy distribution (Fig. 5.13d, no comparable results available) shows, furthermore, a very modest entropy error $(0.002)$ just upstream of the foot of both shock waves. (The smearing of discontinuities in radial direction (Figs. $5.13 \mathrm{c}$ and d) is only due to the grid enlargement in this direction.) The lift and drag value seem to be converged for practical purposes within (again) only a few IDeC-cycles.

For the supersonic test case, we compare our solution with those of Schmidt and Jameson [14] and Veuillot and Vuillot [14]. As grid, they used a $320 \times 64 O$-type grid and a $201 \times 55 C$-type grid, respectively. As lift and drag, they found: $c_{1}=0.5138, \quad c_{\mathrm{d}}=0.1538$ respectively $c_{1}=0.5280, c_{\mathrm{d}}=0.1536$, whereas we found: 
$c_{1}=0.5237, c_{d}=0.1551$. Except for a slight difference in drag and upstream location of the bow shock (Fig. 5.14c), the agreement between our results and the reference results is very good. Our entropy distribution (Fig. 5.14d) shows a very modest entropy error $(0.002)$ just upstream of the airfoil's tail. The same conclusion holds as for the previous test case: with an unadapted and relatively coarse grid, a good solution is obtained.

For the airfoil flows computed on the $128 \times 32$-grid, we needed on an average 5 IDeC-cycles to drive the lift coefficient to within $\frac{1}{2} \%$ of its final value. On the CDC Cyber 205 (single pipe version) on which we performed all our computations, this took us $\sim 100$ s (i.e. $\sim 25 \mathrm{~ms}$ per volume) in scalar mode, and $\sim 50 \mathrm{~s}$ in vector mode. In scalar mode, we obtained the same computational rate per volume for both coarser and finer grids. The convergence rates of both FAS (inner iteration) and IDeC (outer iteration) appear to be independent of the number of volumes on the finest grid (grid-independent).

We did not extensively tune our code for use on vector computers since we did not expect significant accelerations by vectorization. However, for large scale computations, where all data cannot be kept in core, the small number of iteration cycles required (5 IDeC-cycles on an average) results in a small number of out-ofcore data transports. For most Euler codes this is significantly more. Since IO-times rather than CPU-times may be the hampering factor in large scale computations on vector computers, we consider this feature as another advantage of the present method.

\section{CONCLUSiONS}

Studying the behaviour of an iterative defect correction (IDeC) process for various second-order schemes, it appeared that the central and upwind projection scheme lead to divergence for the basic flow problem considered: a transonic airfoil flow with shock. For this flow problem, the other projection schemes; an upwind biased scheme, a superbox scheme, and an upwind scheme supplied with the van Albada limiter (van Albada scheme), all give convergence. The converging schemes all give sharp discontinuities, but they all introduce wiggles, already after the first IDeC-cycle. However, for the van Albada scheme the wiggles disappear within only a few IDeC-cycles. Comparison with the results of other investigators shows that for flows with discontinuities, we obtain solutions of the same good quality with a finest grid which may be twice as coarse (in both directions). For smooth problems the upwind biased, superbox, and van Albada scheme behave in the same way; they all yield solutions of good quality.

For the multigrid computation of second-order accurate flow solutions, IDeC is found to be a very efficient tool. In all cases it converges fast. Both for smooth and non-smooth flow problems, it appeared that it is sufficient to perform only a few IDeC-cycles, each implying only a single multigrid (FAS) cycle.

An important property of the present computational method is that it is completely parameter-free; it needs no tuning of parameters. 


\section{ACKNOWLEDGMENTS}

The author thanks P. W. Hemker for giving valuable suggestions, J. J. Rusch for implementing contour-plotting software, NLR for providing grids, and Vieweg Verlag and AGARD for permitting the use of reference results.

\section{REFERENCES}

1. G. D. van Albada, B. van Leer, and W. W. Roberts, Astron. Astrophys. 108, 76 (1982).

2. K. Böhmer, P. W. Hemker, AND H. J. Stetter, Comput. Suppl. 5, 1 (1984).

3. L. E. Eriksson ANd A. Rizzi, J. Comput. Phys. 57, 90 (1985).

4. W. HaCKBusCh, Multigrid Methods and Applications (Springer-Verlag, Berlin, 1985).

5. P. W. Hemker, in Proceedings, 2nd European Conference on Multigrid Methods, Cologne, 1985, edited by W. Hackbusch and U. Trottenberg (Springer-Verlag, Berlin, 1986).

6. P. W. Hemker and S. P. Spekreisse, Appl. Num. Math. 2, 475 (1986).

7. B. KOREN, Centre for Mathematics and Computer Science Report NM-R8601, 1986; in Proceedings, V. Aerodynamic Seminar, Aachen, 1987.

8. B. VAN LeER, in Lectures in Applied Mathematics Vol. 22, edited by B. E. Engquist et al. (Amer. Math. Soc., Providence, RI, 1985).

9. S. Osher and F. SOlomon, Math. Comput. 38, 339 (1982).

10. A. Rizzi and H. Viviand, Numerical Methods for the Computation of Inviscid Transonic Flows with Shock Waves (Vieweg Verlag, Braunschweig, 1981).

11. S. P. SPEKreisse, in Proceedings, 2nd European Conference on Multigrid Methods, Cologne, 1985, edited by W. Hackbusch and U. Trottenberg (Springer-Verlag, Berlin, 1986).

12. S. P. SPEKreisse, Centre for Mathematics and Computer Science Report NM-R8611, 1986; Math. Comput. 49, 135 (1987).

13. P. K. SWeby, SIAM J. Num. Anal. 21, 995 (1984).

14. H. Viviand, in Test Cases for Inviscid Flow Field Methods, AGARD-AR-211 (AGARD, Neuilly sur Seine, 1985). 